\title{
Renart, récit de la nécessité : une lecture économique de la Branche XVI, « Renart Empereur »
}

\author{
Clément Courteau \\ Université McGill
}

Le Roman de Renart met en scène le premier anti-héros de la littérature française. Ce personnage, qui trahit systématiquement ses amis et camarades pour se nourrir, est l'expression d'un monde où la nécessité est toute-puissante. Omniprésente dans le roman, la méchanceté acquiert alors une valeur progressiste : elle permet au héros de se sustenter à travers une comédie violente faite de duperie et de mensonges. C'est la renardie, forme originale du Roman de Renart, distincte 
des romans courtois ou des chansons de geste. Appelé "roman », le livre s'éloigne effectivement de ce genre par le nombre restreint de topoï qui en constituent les multiples branches, abondamment repris à l'intérieur de l'œuvre mais qui ne se retrouvent pas en dehors. Le fossé intertextuel qui isole le Renart trace les bornes d'une matière qui n'a pas encore accédé au libre jeu des répétitions et variations du roman courtois parodique. Nous tenterons ici d'exposer, à travers une lecture de la BrancheXVI, où se côtoient renardie et parodie romanesque, ce que signifie la forme particulière du Renart et le rapport qu'y entretient la méchanceté avec la représentation du besoin.

\section{Un récit mal construit : structure de la Branche XVI}

Dans l'appareil critique de l'édition de la Pléiade du Roman de Renart, Armand Strubel décrit la Branche XVI, "Renart Empereur ", comme un "récit mal construit » où « les épisodes s'enchaînent et ne sont pas reliés entre eux par une composition nécessaire. On pourrait par exemple faire l'économie de l'épisode de Renart face aux mûres et passer directement au suivant sans que l'architecture de l'ensemble ne soit bouleversée. » (p. 1246, nous soulignons). Strubel s'inscrit ainsi dans une tradition critique qui décrie cette branche pour son «style traînant et lent partout » (Martin, ibid., p. 1249) qui en fait le récit «le plus long et peut-être le moins attrayant» (Foulet, ibid., p. 1247) du roman. La branche est perçue comme une sorte de fourre-tout où, après des aventures somme toute classiques et arbitrairement agencées, l'auteur, en mettant le 
héros en scène dans l'univers courtois qui constitue sa seconde partie, «n'a produit qu'une imitation maladroite des chansons de geste et des romans d'aventures » (Bossuat, ibid., p. 1252). À la fin, le récit ayant délaissé la matière habituelle de la renardie, on se trouve dans un univers simplement courtois : "Renart n'est plus qu'un homme, qu'un chevalier. » (Martin, ibid., p. 1252)

Toutefois, parce que "Renart Empereur» convoque à la fois renardie et parodie, la branche nous paraît appeler une lecture davantage herméneutique que normative. Si, en effet, elle n'est pas la représentante la plus typique ni la plus mémorable des aventures de Renart et d'Isengrin, elle a toutefois la particularité de faire le pont entre deux univers de fiction aux prémisses et aux conventions très différentes. Alors que la renardie est un récit tissé autour de la misère et du besoin ${ }^{1}$, le roman courtois, de même que son pendant négatif parodique, se déroule dans un univers idéalisé, où l'humain est émancipé des conditions matérielles d'existence. La cohabitation des deux univers, alors, nous semble hautement significative. En postulant une cohérence interne dans la construction de la branche, on s'affairera ici à observer sa mécanique pour découvrir comment s'articulent l'univers romanesque et celui de la renardie. D'ailleurs, la matérialité de l'œuvre telle qu'elle nous a été transmise pose un témoignage en défaveur de la tendance de l'arbitraire de la composition : elle se retrouve telle quelle, sans variante notable, dans presque tous les manuscrits du Renart (Strubel, 1998, p. 1246). Il est difficile de croire que, si la branche avait effectivement été aussi décousue et arbitrairement construite qu'on le décrit, les clercs l'auraient reproduite aussi

1 Voir à cet effet l'excellent article de Sarah Gordon (2007), «Hungry like a wolf, sly like a fox », qui montre comment la faim est le moteur narratif du Roman de Renart, prémisse que nous intégrons ici à notre analyse. 
fidèlement de manuscrit en manuscrit. Que le texte, comme le remarque Strubel, ait « résisté à toutes les tentations de partition et de dépeçage » (ibid.), nous conforte dans notre choix et c'est donc pour prendre le contre-pied du jugement de Strubel que nous analyserons la Branche XVI sous l'angle de la nécessité, comprise sous son acception logique, comme rapport d'obligation réciproque entre les divers éléments dans l'architecture du récit, et économique, comme rapport fondamental de l'être humain aux besoins vitaux qui constitue le moteur de la renardie. Ainsi, la méchanceté de Renart passe d'un comportement intéressé (dans la renardie) à un comportement gratuit et, pour ainsi dire, généreux (dans la parodie).

\section{Renart face à la nécessité}

L'ouverture de la branche est classique au Renart. Elle reprend l'anti-reverdie qui ouvre plusieurs autres branches du roman (VI, XII notamment), où l'on voit, avec le printemps qui renaît, la faim tenailler Renart et sa famille : «Ce fu en la douce saison / Que cler chantent li oisillon / Por le tens qui st nés et pur / Que Renars fu dedens les murs / De Malpertuis son fort manoir, / Mais molt ot le cuer tristre et noir / Por sa vïande qui li lasche. / Durement s'estent en soufface, / De fain li duellent li bouel. » $\left(R E^{2}\right.$, v. 1-23) Avec les plaintes de Rovel, de Malebranche et de

\footnotetext{
2 Nous désignons par «Renart Empereur » la Branche XVI du manuscrit $\mathrm{H}$ du Roman de Renart. Nous nous référons à la version du texte proposée par Armand Strubel dans Bibliothèque de la Pléiade. Le texte sera désigné par $R E$.

${ }^{3}$ Pour plus de précision, nous ferons référence, plutôt qu'aux pages, directement aux vers de la Branche XVI, correspondant aux pages 563 à 645 de l'édition de la Pléiade.
} 
Percehaie, l'horizon d'attente d'un lecteur du Renart est respecté en tout point: la faim, au début de l'épisode, force le héros à tenter des aventures pour, après ruses et violences, apaiser sa faim. D'un ventre vide à un ventre plein et ainsi de suite : le cycle du besoin gouverne la progression des épisodes et l'inévitable retour à la case départ qui les fait se succéder. À l'intérieur de ceux-ci, la redondance des motifs de la renardie imite cette circularité. La situation d'Hermeline, par contre, représente un élément inédit: on apprend qu'elle est " enchainte » et qu'elle a " ensi grant fain, / Si en cuic perdre [son] enfant. » (RE, v. 22-25) La nouveauté de la grossesse, au tout début de la branche, brise la cyclicité ordinaire du besoin : l'avenir de Renart mis en péril par la famine qui menace sa descendance, c'est la survie même de sa geste qui dépendra de la quête alimentaire. C'est ainsi que la Branche XVI s'ouvre à l'Événement au sens fort, celui qui brise le cycle du retour et ouvre la possibilité d'une transformation profonde. Et effectivement, avec la mort d'Hermeline et l'ennoblissement de sa famille, la situation de Renart sera définitivement transformée.

Renart part donc pour une quête de nourriture à haut degré de senefiance. Les péripéties de son chemin forment une sorte de parcours initiatique, où les éléments habituels du récit sont suspendus et où les motifs usuels du Renart sont transformés. On voit d'abord Isengrin lui témoigner une vive amitié, même après s'être fait piéger par Renart. L'inimitié des deux personnages, constitutive du roman et explicitée dans les Enfances Renart où on voit, dès leur création, le loup et le renard se livrer une bataille mortelle, se trouve suspendue. Isengrin et Hersent invitent Renart à un banquet somptueux, lors duquel on peut voir dans leur comportement courtois 
réciproque ${ }^{4}$ la prolepse de la fin de la branche, où le récit, abandonnant la renardie, s'anthropomorphise et passe à la chevalerie. Dans cette camaraderie irréelle, qui permettra plus tard à Renart d'usurper le trône ${ }^{5}$, il y a un élément de grâce qui, en brisant l'attente principale du lecteur, contribue à l'effet d'étrangeté de la branche et la présente comme la branche de la réconciliation, celle par laquelle on pourrait enfin sortir du cycle de violence qui habite le Renart.

L'épisode des mûres survient aussitôt le départ de Renart de chez Isengrin, départ hâté par le rappel de la situation d'exception qui le force à poursuivre son chemin («Molt prie Dieu que il l'avoit / En tel lieu ou vïande truisse / Que a sa feme porter puisse / Que il laissa ençainte et grosse. » (RE, v. 264268). Cet épisode, dont se sert Strubel pour exemplifier la composition arbitraire de la branche, disant qu'on pourrait en faire l'économie «sans que l'architecture de l'ensemble ne soit bouleversée », représente, Strubel le reconnaît lui-même, un autre moment unique du Renart, «la seule [scène] de tout le Roman où nous voyons le héros, seul, mis en échec par les forces de la nature» (1998, p.1247). En effet, lors de cet épisode, Renart, qui aperçoit un buisson de mûres, tente, sans succès, de les atteindre et finit par passer son chemin. Le caractère double de la nature, à la fois désert et corne

\footnotetext{
${ }^{4}$ On peut observer le meilleur exemple de ce comportement lorsque Renard tente de prendre congé d'Isengrin : «Ha, sire, dist Renars, merci. / Je ne puis plus demorer ci, / Car j'ai a faire en austres leus. / - N'en irés pas, ce dist li leus, / Hui ne demain, foi que vous doi. / - Sire, dist Renars, par ma foi / Je ne demorroie por rien, / Mais de verité saciés bien / Que au plus tots que je porrai, / Ci iluec a vous revenrai. » $(R E$, v. 242-254)

5 C'est en effet Isengrin qui recommandera Renart à Noble pour la garde de son château : «Sire, [...] Tous li mieudrez que jou i voi / Et que sache eslire entre nous, / Ce est Renars, foi que doi vous; / Hardis est et de fier coraige / Et molt a en lui vasselaige / Et si est bien enparentés. » (RE, v. 1911-1917)
} 
d'abondance, apparaît dans la construction de la découverte des mûres. D'abord, Renart, qui après avoir imploré Dieu de l'envoyer dans un lieu où il trouverait à manger, arrive à " une fosse / qui molt estoit parfons et grans» (RE, v. 268-269). Naturellement, vu son espoir de voir sa prière exaucée, il s'y arrête «longuement [...] por esgarder que deens ot» $(R E$, v. 272-273) Mais «quant assés esgardé ot, / vit qu'ele fu de ronches plainne / si durement que a grant painne / i Paroit il se ronces non » (RE, v. 274-277). D'abord, il y a le vide du fossé, profond et grand. Puis, à force de regarder, quand on a assez regardé, le vide se révèle rempli, mais rempli de ronces, plantes agressives et non comestibles. C'est au troisième regard, quand «tant a esgardé environ», que Renart «vit que meures i ot tant / que onques mais en son vivant/ n'en avoit tant veü ensanble » (RE, v. 278-281). L'abondance n'apparaît qu'à la fin $\mathrm{du}$ parcours d'observation, non pas comme une gratuité généreuse, immédiatement accessible, mais comme un élément lointain, à la réalité incertaine, situé par-delà le voile de la misère, représentée ici par les ronces. C'est à peine si on aperçoit les mûres, et leur abondance n'est qu'illusoire, car elles sont hors d'atteinte. Pour les attraper, même la ruse de Renart fait défaut. Ce qui fonctionne habituellement avec les autres animaux ou avec les villageois se révèle complètement inefficace face à la nature : "Je suis fox qui ici demeure, / dit Renart en quittant les lieus, / que je ne menjus nule meure. / N'en manjai lonc tans a passé / Et par mon chief je l'ai voué / Que je n'en menjerai jamais. » $(R E$, v. 331-335) L'accès à l'abondance, relégué à un lointain passé, est définitivement barré. Ici, Renart fait l'expérience directe de la nécessité qui gouverne partout ailleurs ses actions, bien qu'indirectement. Habituellement, les ressources, produit du travail des animaux 
ou des humains, sont relativement faciles à acquérir pour lui, car elles se trouvent dans un contexte social où la tromperie est fonctionnelle. Cette confrontation avec la nature le met en rapport direct, sans intermédiaire social, sans lois à briser ni confiance à tromper, avec la Nécessité, représentée par le barrage infranchissable qui le sépare des mûres. On ne peut tromper la nature, on ne peut ruser contre la nécessité : elle commande une résignation complète, une obéissance totale.

Immédiatement après avoir passé son chemin, Renart rencontre Roonel dans un épisode qui, selon Strubel, « constitue le maillon le plus faible de cette chaîne : le conteur semble faire du remplissage » (p. 1247). Roonel, qui gît au pied d'un arbre, «avoit esté batus / d'un vilain si fort et frapés / A poi n'avoit esté tués; / Ne pot movoir ni ié ne main» ( $R E$, v. 344-347). Renart se dirige vers lui, pour le prendre dans un autre de ses engins cruels, respectant les motifs usuels de la renardie. Toutefois, ce qui nous intéresse ici, c'est l'incursion du souvenir de la nécessité dans la mécanique de la renardie. En se dirigeant vers le chien, juste avant de bondir sur lui, on précise que Renart «mout est dolans et coreciés / des meures ou il ot failli » $(R E$, v. 350-351). Le premier épisode de méchanceté pure de la Branche XVI, si on exclut un tour bénin joué à l'ami Isengrin plus tôt, a lieu tout de suite après l'échec des mûres et, pour encore ramener l'attention sur cette filiation, le conteur le rappelle juste avant le déploiement de la mécanique narrative bien connue des pièges de Renart. La violence, dans la Branche XVI, survient directement après la confrontation avec le vide de la nature et le barrage des ronces de la nécessité.

Le caractère rebelle de Renart trouve dans les mûres son asymptote : il ne pourra jamais triompher complètement de la 
nécessité, jamais atteindre la satisfaction complète. À ce sujet, Luke Sunderland parle, pour décrire l'amoralité rigoureuse du Roman de Renart, d'une éthique de la pulsion: "The drive is a constant, pulsating force that can never be satisfied and that is not subject to bodily rhythms. The purpose of the drive is not satisfaction throught the attainment of any particular object, but rather the very process of circling the object. ${ }^{6}$ (2010, p. 159) La pulsion est ce qui demeure une fois le besoin satisfait, le surplus utopique caché derrière la barrière des ronces et à jamais inaccessible. Ce qui avait été jusqu'ici une épopée de la faim devient un roman du désir. Et c'est exactement la trajectoire que prend la BrancheXVI, de la renardie à la parodie, du slapstick alimentaire au jeu d'intertextualité libre et circulaire. "The real purpose of the drive, poursuit Sunderland, is not some mystical goal of full satisfacion, but to return to its circular path, and the real source of enjoyment is the repetitive movement of this closed circuit. $\gg^{7}$ (p. 159) Mais ce circuit de la pulsion ne peut exister pour soi que si la nécessité est dépassée. En effet, la faim, parce qu'elle oblige la réalisation des désirs, est une expérience anticivilisationnelle radicale. Elle ne permet aucune tension, aucune jouissance. Renart ne peut obéir correctement à la pulsion qu'à deux conditions. Il doit d'abord avoir fait l'expérience de l'objet inaccessible, qui barre la route à toute

\footnotetext{
6 « La pulsion est une force pulsatile constante qui ne peut jamais être satisfaite et qui n'est pas sujette aux rythmes corporels. La raison dêtre de la pulsion n'est pas l'atteinte d'un objet en particulier, mais plutôt le processus même de gravitation autour de l'objet. » (Nous traduisons)

7 «La véritable raison d'être de la pulsion n'est pas l'atteinte mystique d'une satisfaction totale, mais bien le retour à son chemin circulaire en circuit fermé. C'est ce mouvement répétitif qui est la véritable source du plaisir. » (Nous traduisons)
} 
transcendance. Une fois cette condition remplie, il peut déployer sa violence gratuitement, sans attendre de gains alimentaires, comme l'illustre le piège inutile qu'il tend à Roonel.

\section{La parodie, récit émancipé}

La seconde condition pour entrer pleinement dans l'utopie pulsionnelle-textuelle de la chevalerie, c'est l'émancipation complète du besoin, ce par quoi se conclut justement la première partie de la Branche XVI : le vol d'un épervier, qui va chasser à sa place et beaucoup plus efficacement que lui, permet à Renart de capturer des canards autant qu'il en veut $(R E$, v. 1554). Cet outil aristocratique automatise son rapport à la faim, comblée à mesure qu'elle survient. «Qu'iroie lonc conte tenant? » (RE, v. 1591), conclut le narrateur, « quatre anes prist en un tenant» (RE, v. 1592 et suivants) Pourquoi, en effet, continuer le récit lorsque la faim est battue, lorsque la nécessité disparaît? Comment la renardie pourra-t-elle se poursuivre après la disparition de ses bornes usuelles ? Parce que Renart a fait l'apprentissage de la nécessité brute de la nature, qu'il a connu à la fois la violence du besoin et un objet de désir inaccessible, il a finalement développé une technique pour se libérer $\mathrm{du}$ besoin et exister librement dans un univers pulsionnel dépourvu de rapport à la nécessité. C'est cet autre monde où le conteur dirige ce héros qui permet de poursuivre le récit une fois l'histoire de Renart terminée pour de bon. Mais il se poursuit par l'abandon de la renardie, du récit animalier, par l'anthropomorphisme total de ses personnages, décrié par 
les critiques de la branche comme étant une transgression des règles du Renart. Après l'épisode de l'épervier, Renart se bat en duel contre Tardif le limaçon, dans un combat de chevaliers classique, avec lances et chevaux, épées et boucliers. Sans ruse, par la simple prouesse, Renard tue Tardif comme un chevalier en tue un autre : " quant Renars le vit cheü / vers lui cort, si li tolt l'escu, / sel fiert dou tabo liés l'oreille / que la teste li fist vermeille. / Tout le vis li a escorchié. / Lors li a tolu son espié [...] / Si li lance par mi le cors; / mort l'a, puis monte, si s'en vait.» (RE, v. 1633-1642) Pour un lecteur du Renart, le contraste est frappant avec les scènes de bataille habituelles, où les animaux souffrent toujours à même leur corps animal, leurs blessures accompagnées de touffes de poil arrachées, de queues coupées ou de museau écorché8. Ici, Tardif, la bête la moins anthropomorphe du roman, saigne de la tête et meurt en chevalier. Le roman a changé de base.

À la lumière de cette transformation, les différents moments de la première partie de la branche apparaissent comme le produit d'une composition nécessaire, qui permet de suivre point à point Renart sur le chemin de la transformation. Cette nécessité de la composition se double de la figuration de la nécessité dans le motif des mûres inaccessibles, seule confrontation de Renart avec la nature. Une fois la faim apaisée par le vol de l'épervier, Renart s'émancipera de sa forme et de son univers pour parvenir dans un monde courtois truffé de

\footnotetext{
8 Voir la déconfiture de Brun l'Ours à la branche X, v. 642-652 : « Taste et retaste et tire et sache, / Estent la pel, rompent les vaines, / Tant a sachié que a grant painnes / Fant li cuirs et la teste qasse; / Mes del sanc i lessa grant masse, / La piau des piez et de la teste. / Onc nus ne vit si lede beste : / Le sans li coule du musel, / En tot son vis n'a tant de pel / Dont l'en peüst fere une borse. / Einssi s'en va le filz a l'orse $[\ldots]$. »
} 
jeux parodiques et intertextuels. Pour mieux rappeler la condition de possibilité de ce tournant ludique, au début de la partie courtoise de la branche, on dépeint Renart chevauchant sont faucon à la main : "son faucon desis son puing tint / Molt resemble bien home apert » $(R E$, v. 1688), « desus son puing le faucon porte, / En aler forment se deporte » (RE, v. 1709) Celui qui était parti en quête pour empêcher la disparition de son avenir a finalement trouvé, par l'émancipation face à la nécessité, un autre monde où s'investir. Il peut alors briser le cycle de la faim, la perpétuation de l'animalité. Les morts d'Hermeline et de l'enfant qu'elle portait viennent confirmer cette transformation irrévocable: il n'y a pas de retour dans l'univers animalier du besoin. Dès qu'il apprend la nouvelle, Renart transfère sa lignée à la cour : «Biaus filz, mais alés tote voie / arriere, si ne demorés / et vous deus freres m'amenés / a la court Noblez li lions. / Tous trois chevaliers fos ferons. » $(R E$, v. 1763-1740) Dans le monde courtois, la méchanceté de Renart peut sortir de l'exiguïté narrative des scènes de renardie pour gagner le grand monde intertextuel des romans de chevalerie.

Dans son étude sur l'intertextualité de la deuxième partie de la Branche XVI, Robert Bellon montre « l'intention parodique plus large, globalisante si l'on peut dire » qui y règne :

Le conteur réécrit à sa façon, tout en l'intégrant dans un récit à tonalité épique (guerre contre les Païens, puis siège du château du roi) tout un épisode bien connu de la saga arthurienne : la révolte de Mordret et l'usurpation du trône par celui à qui le roi avait confié la régence du royaume durant son absence. (2008, p. 1-2)

En effet, dans la guerre contre les païens qui se prépare, le conteur reprend des motifs épiques comme le catalogue ${ }^{9}$ et

${ }^{9}$ Les deux armées ennemies sont présentées à tour de rôle, d'abord l'armée païenne, formée d'animaux exotiques (v.1770-1781), ensuite l'armée 
les combats sanguinaires (« Tyecelins tint ou poing l'espee / qui ot le branc clerc et moulu. / S'a un escorpïon feru, / la teste li cope et les piés.» (RE, v. 2090-2093), dont la grandeur est systématiquement diminuée par la nature animale des combattants, comme en témoigne l'entrée en guerre majestueuse de Frobert le grillon : «Quant Frobers li gresillonz saut, 0 lui de sa gent a plenté. / Molt par sont cil mal atrapé, / Plus de vint mile ocis en ont. » (RE, v. 2214-2217) Tandis que les insectes triomphent sur le champ de bataille, Renard, resté à la cour pour veiller sur le palais, devient un hybride de Lancelot et de Mordret par la relation d'amour qu'il entretient avec Fière, puis par la trahison qu'il prépare : «Renart qui fu molt mal senés / fu remés avoec la roïne / Qui l'aimoit d'amoureuse treine / et longement l'avoir amee », (RE, v. 2001-2005), "Un petitet s'est porpensés / A soi meïsmes et a dit/ Que se Damediex li aït, / Empereres sera et rois. » (RE, v. 2304-2308) Ce second passage, qui convoque les moments les plus forts du cycle arthurien (la trahison de Mordret, l'adultère de Lancelot et de Guenièvre), est introduit par une formule de séparation typique des romans en prose : « Ci luex vous lairons dou roi, / $\mathrm{Si}$ vous redirons de Renart.» (RE, v. 2300-2301) Le héros de la renardie, une fois transposé dans l'univers parodique du roman courtois, incarne ses plus sombres figures. Sa méchanceté brute, raffinée par l'intertexte arthurien, devient, en investissant la figure de Mordret, un élément clef du déroulement narratif du cycle arthurien et, par là, accède à un statut tout autre que la tromperie du Renart.

chrétienne, formée d'animaux conventionnels (v. 1794-1818). Le contraste faunique parodie ici l'esthétique manichéenne des chansons de geste, où les païens sont dépeints comme le négatif des chrétiens. 


\section{La fin de la renardie}

La véritable fin du Roman, ce n'est pas la Mort Renart, impuissante à clore le récit de l'anti-héros qui ruse même contre son propre décès, mais l'émancipation vis-à-vis du besoin, qui permet l'humanisation du héros et sa translation dans l'univers de la chevalerie. Renart, qui délaisse le monde animal du besoin, découvre en même temps celui, humain, de la pulsion. Le surplus pulsionnel qu'il a découvert avec les mûres, objet inatteignable autour duquel on ne peut que tourner, dans un mouvement répétitif en circuit fermé (Sunderland, voir plus haut), lui permet de continuer d'exister malgré l'extinction de sa faim. Textuellement, on pourrait appeler parodie ce mouvement circulaire autour de l'objet. La parodie qui n'atteint jamais directement l'essence du récit, qui ne s'empare pas de son identité, mais reste toujours en périphérie et propose des variations et des revirements, des jeux intertextuels sans fin. Le mouvement parodique, sans but nécessaire, est la tromperie de Renart utilisée non comme moyen mais comme fin, la ruse pour la ruse. Elle dévoile ainsi le monde idéalisé de la cour non pas comme le royaume du bien et la fin de l'histoire, mais comme un lieu permettant la variation libre du texte sur ses divers thèmes. Ainsi, Renart Empereur, par son éloignement soudain des canons du Roman, met en lumière une caractéristique fondamentale du Roman de Renart, à savoir que cette œuvre, qui s'élabore autour du thème de la faim, est l'écriture de la nécessité, tandis que le roman représente celle de la contingence. Ainsi, tout comme la nécessité est le passé trouble de la liberté, héritière qui en porte encore les stigmates, le Renard est l'autrefois du roman. 


\section{Bibliographie}

BELLON, Roger. (2008), «Renart empereur: Le Roman de Renart, ms. H, Branche XVI : une réécriture renardienne de La Mort le roi Artu?», Cahiers de recherches médiévales (XIIIe-XVe siècles), vol. 15, no 1, p. 3-17.

GORDON, Sarah. (2007), Culinary Comedy in medieval French literature, Lafayette, Purdue University Press.

STRUBEL, Armand (dir.). (1998), Le Roman de Renart, Paris, Gallimard, coll. " Bibliothèque de la Pléiade ».

SUNDERLAND, Luke. (2010), Old French Narrative Cycles: Heroism between ethics and morality, Cambridge, Brewer, coll. « Gallica ».

\section{Résumé}

La Branche XVI du Roman de Renart est souvent décriée pour une construction qui serait arbitraire. Toutefois, l'interprétation des scènes qui se succèdent au long de la première partie révèle un récit organisé, centré sur la Nécessité et la quête de Renart pour s'en émanciper. C'est dans ce rapport avec elle qu'apparaît la nature véritable de la méchanceté de Renart : une extension du besoin auquel le héros est acculé. Ce n'est qu'en s'émancipant de cette nécessité que le héros accèdera au roman, forme dans laquelle sa méchanceté deviendra, plus qu'une comédie redondante, le moteur de l'action d'un grand drame romanesque médiéval. 


\begin{abstract}
The Sixteenth Branch of the Roman de Renart, often criticised for its supposed arbitrary construction. But with the interpretation of the scenes that constitute the first part of the Branch, we can see that it is in fact an organized narrative centered around Necessity, and Renart's quest to break away from it. We can then discover what lies underneath Renart's cruelty: an extension of his relationship with the most basic needs. Only when he can be outgrow necessity, can Renart gain access to the novel, a literary form where his cruelty can become, more than a redundant slapstick comedy, the agent of a great medieval drama.
\end{abstract}

